\title{
SOME PROPERTIES OF THE REPRESENTATION CATEGORY OF TWISTED DRINFELD DOUBLES OF FINITE GROUPS
}

\author{
Deepak Naidu \\ Received: 21 June 2020; Accepted 7 August 2020 \\ Communicated by Sait Halıcıoğlu
}

\begin{abstract}
A criterion for a simple object of the representation category $\operatorname{Rep}\left(D^{\omega}(G)\right)$ of the twisted Drinfeld double $D^{\omega}(G)$ to be a generator is given, where $G$ is a finite group and $\omega$ is a 3-cocycle on $G$. A description of the adjoint category of $\operatorname{Rep}\left(D^{\omega}(G)\right)$ is also given.
\end{abstract}

Mathematics Subject Classification (2020): 18M20

Keywords: Drinfeld double, quantum double

\section{Introduction}

Modular tensor categories arise in several diverse areas such as quantum group theory, vertex operator algebras, and rational conformal field theory. Let $G$ be a finite group, let $D(G)$ denote the Drinfeld double of $G$, a quasi-triangular semisimple Hopf algebra, and let $\operatorname{Rep}(D(G))$ denote the category of finite-dimensional complex representations of $D(G)$. The category $\operatorname{Rep}(D(G))$ is a modular tensor category [1], and it is perhaps the most accessible constructions of a modular tensor category. As such, it is desirable to have a thorough understanding of this category. In this paper, we make a contribution towards this goal. The category $\operatorname{Rep}(D(G))$ is equivalent to the $G$-equivariantization of $\mathrm{Vec}_{G}$, and it is also equivalent to the center $\mathcal{Z}\left(\operatorname{Vec}_{G}\right)$ of the tensor category $\operatorname{Vec}_{G}$ of finite-dimensional $G$-graded complex vector spaces.

In the papers [3,4], R. Dijkgraaf, V. Pasquier, and P. Roche introduce a quasitriangular semisimple quasi-Hopf algebra $D^{\omega}(G)$, often called the twisted Drinfeld double of $G$, where $\omega$ is a 3-cocycle on $G$. When $\omega=1$ this quasi-Hopf algebra coincides with the Drinfeld double $D(G)$ considered above. The category $\operatorname{Rep}\left(D^{\omega}(G)\right)$ of finite-dimensional complex representations of $D^{\omega}(G)$ is a modular tensor category. Analogous to the $\omega=1$ case, the category $\operatorname{Rep}\left(D^{\omega}(G)\right)$ is equivalent to the $G$-equivariantization of $\operatorname{Vec}_{G}^{\omega}$, and it is also equivalent to the center $\mathcal{Z}\left(\operatorname{Vec}_{G}^{\omega}\right)$ of the tensor category $\operatorname{Vec}_{G}^{\omega}$ of finite-dimensional $G$-graded complex vector spaces with associativity constraint defined using $\omega$. Every braided group-theoretical fusion 
category is equivalent to a full fusion subcategory of some $\operatorname{Rep}\left(D^{\omega}(G)\right)$, and all such subcategories were parametrized in the paper [12].

This paper contains two main results, stated below. The first gives a criterion for a simple object of $\operatorname{Rep}\left(D^{\omega}(G)\right)$ to be a generator, and the second gives a description of the adjoint category of $\operatorname{Rep}\left(D^{\omega}(G)\right)$.

Theorem. Let $G$ be a finite group, let $\omega$ be a normalized 3-cocycle on $G$, and let $(a, \chi)$ be a simple object of $\operatorname{Rep}\left(D^{\omega}(G)\right)$. Then $(a, \chi)$ is a generator of $\operatorname{Rep}\left(D^{\omega}(G)\right)$ if and only if the following two conditions hold.

(a) The normal closure of a in $G$ is equal to $G$.

(b) For all $b \in Z(G)$ and $\chi^{\prime} \in \operatorname{Irr}_{\beta_{b}}(G)$, if $\chi(b) \chi^{\prime}(a)=\operatorname{deg} \chi \operatorname{deg} \chi^{\prime}$ (equivalently, $(a, \chi)$ and $\left(b, \chi^{\prime}\right)$ centralize each other $)$, then $b=e$ and $\chi^{\prime}=1$.

Theorem. Let $G$ be a finite group, and let $\omega$ be a normalized 3-cocycle on $G$. Then

$$
\operatorname{Rep}\left(D^{\omega}(G)\right)_{\mathrm{pt}}=\mathcal{S}\left(Z_{\omega}(G),[G, G], B\right)
$$

and

$$
\operatorname{Rep}\left(D^{\omega}(G)\right)_{\mathrm{ad}}=\mathcal{S}\left([G, G], Z_{\omega}(G),\left(B^{\mathrm{op}}\right)^{-1}\right)
$$

where $B: Z_{\omega}(G) \times[G, G] \rightarrow \mathbb{C}^{\times}$is the $G$-invariant $\omega$-bicharacter defined in Lemma 4.4 .

\section{Organization:}

In Section 2, we recall basic facts about the modular tensor category $\operatorname{Rep}\left(D^{\omega}(G)\right)$. In Section 3, we prove the first theorem above, and in Section 4, we prove the second theorem.

\section{Convention and notation:}

Throughout this paper we work over the field $\mathbb{C}$ of complex numbers. The multiplicative group of nonzero complex numbers is denoted $\mathbb{C}^{\times}$. Let $G$ be a finite group. The identity element of $G$ is denoted $e$, and the center of $G$ is denoted $Z(G)$. For any character $\chi$ of $G$, the degree of $\chi$ is denoted $\operatorname{deg} \chi$, the complex conjugate of $\chi$ is denoted $\bar{\chi}$, and the kernel of $\chi$ is denoted $\operatorname{Ker} \chi$. Let $\mu$ be a 2-cocycle on $G$ with coefficients in $\mathbb{C}^{\times}$. The set of irreducible $\mu$-characters of $G$ is $\operatorname{denoted~} \operatorname{Irr}_{\mu}(G)$. When $\mu=1$, we write $\operatorname{Irr}(G)$ instead of $\operatorname{Irr}_{1}(G)$. Finally, the coboundary operator on the space of cochains of $G$ with coefficients in $\mathbb{C}^{\times}$is denoted $d$. 


\section{Drinfeld doubles of finite groups}

Let $G$ be a finite group. As stated earlier, the category $\operatorname{Rep}(D(G))$ of finitedimensional representations of the Drinfeld double $D(G)$ is a modular tensor category [1]. The simple objects of $\operatorname{Rep}(D(G))$ are in bijection with the set of pairs $(a, \chi)$, where $a$ is a representative of a conjugacy class of $G$, and $\chi$ is an irreducible character of the centralizer $C_{G}(a)$ of $a$ in $G$. The $S$-matrix and the $T$-matrix of $\operatorname{Rep}(D(G))$ are square matrices indexed by the simple objects of $\operatorname{Rep}(D(G))$, and are given by the following formulas $[1,2]$.

$$
\begin{aligned}
& S_{(a, \chi),\left(b, \chi^{\prime}\right)}=\frac{1}{\left|C_{G}(a)\right|\left|C_{G}(b)\right|} \sum_{g \in G(a, b)} \bar{\chi}\left(g b g^{-1}\right) \overline{\chi^{\prime}}\left(g^{-1} a g\right), \\
& T_{(a, \chi),\left(b, \chi^{\prime}\right)}=\delta_{a, b} \delta_{\chi, \chi^{\prime}} \frac{\chi(a)}{\operatorname{deg} \chi},
\end{aligned}
$$

where $G(a, b)$ denotes the set $\left\{g \in G \mid a g b g^{-1}=g b g^{-1} a\right\}$.

Let $\omega: G \times G \times G \rightarrow \mathbb{C}^{\times}$be a normalized 3-cocycle. Then

$$
\omega(b, c, d) \omega(a, b c, d) \omega(a, b, c)=\omega(a b, c, d) \omega(a, b, c d)
$$

for all $a, b, c, d \in G$, and $\omega(a, b, c)=1$ if $a, b$, or $c$ is the identity element. Replacing $\omega$ by a cohomologous 3-cocycle, if necessary, we may assume that the values of $\omega$ are roots of unity.

For each $a \in G$, define a function $\beta_{a}: G \times G \rightarrow \mathbb{C}^{\times}$by

$$
\beta_{a}(x, y)=\frac{\omega(a, x, y) \omega\left(x, y, y^{-1} x^{-1} a x y\right)}{\omega\left(x, x^{-1} a x, y\right)} .
$$

The 3-cocycle condition on $\omega$ ensures that the relation

$$
\beta_{x^{-1} a x}(y, z) \beta_{a}(x, y z)=\beta_{a}(x y, z) \beta_{a}(x, y)
$$

holds for all $a, x, y, z \in G$. Therefore, for any $a \in G$, the restriction of $\beta_{a}$ to the centralizer $C_{G}(a)$ of $a$ in $G$ is a normalized 2-cocycle, that is,

$$
\beta_{a}(y, z) \beta_{a}(x, y z)=\beta_{a}(x y, z) \beta_{a}(x, y)
$$

for all $x, y, z \in C_{G}(a)$, and $\beta_{a}(x, y)=1$ if $x$ or $y$ is the identity element.

For each $a \in G$, define a function $\gamma_{a}: G \times G \rightarrow \mathbb{C}^{\times}$by

$$
\gamma_{a}(x, y)=\frac{\omega(x, y, a) \omega\left(a, a^{-1} x a, a^{-1} y a\right)}{\omega\left(x, a, a^{-1} y a\right)} .
$$

Direct calculations using the 3-cocycle condition of $\omega$ show that

$$
\frac{\beta_{a}(x, y) \beta_{b}(x, y)}{\beta_{a b}(x, y)}=\frac{\gamma_{x y}(a, b)}{\gamma_{x}(a, b) \gamma_{y}\left(x^{-1} a x, x^{-1} b x\right)}
$$


for all $a, b, x, y \in G$. For all $a \in G$, the functions $\beta_{a}$ and $\gamma_{a}$ are equal when restricted to $C_{G}(a)$. Therefore, we have

$$
\frac{\beta_{a}(x, y) \beta_{b}(x, y)}{\beta_{a b}(x, y)}=\frac{\beta_{x y}(a, b)}{\beta_{x}(a, b) \beta_{y}(a, b)}
$$

for all $a, b \in Z(G)$, and $x, y \in G$.

As stated earlier, the category $\operatorname{Rep}\left(D^{\omega}(G)\right)$ of finite-dimensional representations of the twisted Drinfeld double $D^{\omega}(G)$ is a modular tensor category. The simple objects of $\operatorname{Rep}\left(D^{\omega}(G)\right)$ are in bijection with the set of pairs $(a, \chi)$, where $a$ is a representative of a conjugacy class of $G$, and $\chi$ is an irreducible $\beta_{a}$-character of the centralizer $C_{G}(a)$ of $a$ in $G$. The $S$-matrix and the $T$-matrix of $\operatorname{Rep}\left(D^{\omega}(G)\right)$ are square matrices indexed by the simple objects of $\operatorname{Rep}(D(G))$, and are given by the following formulas [2].

$$
\begin{aligned}
& S_{(a, \chi),\left(b, \chi^{\prime}\right)} \frac{}{\left(\frac{\beta_{a}\left(x, g^{\prime}\right) \beta_{a}\left(x g^{\prime}, x^{-1}\right) \beta_{b}(y, g) \beta_{b}\left(y g, y^{-1}\right)}{\beta_{a}\left(x, x^{-1}\right) \beta_{b}\left(y, y^{-1}\right)}\right)} \bar{\chi}\left(x g^{\prime} x^{-1}\right) \bar{\chi}^{\prime}\left(y g y^{-1}\right) \\
& =\sum_{\substack{g \in C \ell_{G}(a) \\
g^{\prime} \in C \ell_{G}(b) \cap C_{G}(g)}} \\
& T_{(a, \chi),\left(b, \chi^{\prime}\right)}=\delta_{a, b} \delta_{\chi, \chi^{\prime}} \frac{\chi(a)}{\operatorname{deg} \chi},
\end{aligned}
$$

where $g=x^{-1} a x, g^{\prime}=y^{-1} b y$, and $C \ell_{G}(a)$ denotes the conjugacy class of $a$ in $G$.

\section{Tensor generators}

In this section, we give a criterion for a simple object $(a, \chi)$ of $\operatorname{Rep}\left(D^{\omega}(G)\right)$ to be a tensor generator, that is, the full fusion subcategory given by the intersection of all fusion subcategories of $\operatorname{Rep}\left(D^{\omega}(G)\right)$ that contain $(a, \chi)$ is $\operatorname{Rep}\left(D^{\omega}(G)\right)$.

Let $\mathcal{C}$ be a modular tensor category with braiding $c$. Two objects $X, Y \in \mathcal{C}$ centralize each other if

$$
c_{Y, X} \circ c_{X, Y}=\operatorname{id}_{X \otimes Y} .
$$

Let $\mathcal{D}$ be a full (not necessarily tensor) subcategory of $\mathcal{C}$. In the paper [10], M. Müger defined the centralizer of $\mathcal{D}$ in $\mathcal{C}$ as the full subcategory of $\mathcal{C}$, denoted $\mathcal{D}^{\prime}$, consisting of all objects in $\mathcal{C}$ that centralize every object in $\mathcal{D}$. That is,

$$
\operatorname{Obj}\left(\mathcal{D}^{\prime}\right)=\left\{X \in \operatorname{Obj}(\mathcal{C}) \mid c_{Y, X} \circ c_{X, Y}=\operatorname{id}_{X \otimes Y} \text { for all } Y \in \operatorname{Obj}(\mathcal{D})\right\} .
$$

It was shown in [10] that $\mathcal{D}^{\prime}$ is a fusion subcategory, and that if $\mathcal{D}$ is a fusion subcategory, then $\mathcal{D}^{\prime \prime}=\mathcal{D}$; we refer to this result as the double centralizer theorem.

We recall the following result from [11]. 
Proposition 3.1. Let $G$ be a finite group, and let $(a, \chi)$ and $\left(b, \chi^{\prime}\right)$ be simple objects of $\operatorname{Rep}(D(G))$. Then $(a, \chi)$ and $\left(b, \chi^{\prime}\right)$ centralize each other if and only if the following two conditions hold.

(a) The conjugacy classes of $a$ and $b$ commute elementwise.

(b) For all $g \in G, \chi\left(g b g^{-1}\right) \chi^{\prime}\left(g^{-1} a g\right)=\operatorname{deg} \chi \operatorname{deg} \chi^{\prime}$.

Below, we record a special case of the result above.

Proposition 3.2. Let $G$ be a finite group, and let $(a, \chi)$ and $\left(b, \chi^{\prime}\right)$ be simple objects of $\operatorname{Rep}(D(G))$, where $b$ lies in the center of $G$, so that $\chi^{\prime} \in \operatorname{Irr}(G)$. Then $(a, \chi)$ and $\left(b, \chi^{\prime}\right)$ centralize each other if and only if the following holds.

(i) $\chi(b) \chi^{\prime}(a)=\operatorname{deg} \chi \operatorname{deg} \chi^{\prime}$.

If $b=e$ or $\chi=1$, then the condition above is equivalent to the condition

(i') $a \in \operatorname{Ker} \chi^{\prime}$

Proof. If $b=e$ or $\chi=1$, then the equality in condition (i) is equivalent to the equality $\chi^{\prime}(a)=\operatorname{deg} \chi^{\prime}$, which is equivalent to condition $\left(\mathrm{i}^{\prime}\right)$.

Suppose that $(a, \chi)$ and $\left(b, \chi^{\prime}\right)$ centralize each other. Putting $g=e$ in condition (b) of Proposition 3.1, we get $\chi(b) \chi^{\prime}(a)=\operatorname{deg} \chi \operatorname{deg} \chi^{\prime}$, which is condition (i).

Conversely, suppose that condition (i) holds. Since $b$ lies in the center of $G$, we know that $C_{G}(b)=G$ and $\chi^{\prime}$ is a character of $G$. Condition (a) of Proposition 3.1 clearly holds. For all $g \in G$, we have $\chi\left(g b g^{-1}\right) \chi^{\prime}\left(g^{-1} a g\right)=\chi(b) \chi^{\prime}(a)$, since $b$ is in the center of $G$ and $\chi^{\prime}$ is a class function on $G$. By supposition, $\chi(b) \chi^{\prime}(a)=$ $\operatorname{deg} \chi \operatorname{deg} \chi^{\prime}$, and so condition (b) of Proposition 3.1 holds. Hence $(a, \chi)$ and $\left(b, \chi^{\prime}\right)$ centralize each other.

Theorem 3.3. Let $G$ be a finite group, and let $(a, \chi)$ be a simple object of $\operatorname{Rep}(D(G))$. Then $(a, \chi)$ is a generator of $\operatorname{Rep}(D(G))$ if and only if the following two conditions hold.

(a) The normal closure of a in $G$ is equal to $G$.

(b) For all $b \in Z(G)$ and $\chi^{\prime} \in \operatorname{Irr}(G)$, if $\chi(b) \chi^{\prime}(a)=\operatorname{deg} \chi \operatorname{deg} \chi^{\prime}$ (equivalently, $(a, \chi)$ and $\left(b, \chi^{\prime}\right)$ centralize each other $)$, then $b=e$ and $\chi^{\prime}=1$.

Proof. By the double centralizer theorem, the simple object $(a, \chi)$ is a generator of $\operatorname{Rep}(D(G))$ if and only if the only simple object that centralizes $(a, \chi)$ is the trivial simple object $(e, 1)$.

Suppose that conditions (a) and (b) in the statement of the theorem hold, and let $\left(b, \chi^{\prime}\right)$ be a simple object of $\operatorname{Rep}(D(G))$ that centralizes $(a, \chi)$. By Proposition 3.1 , the conjugacy classes of $a$ and $b$ commute elementwise; combining this fact with 
condition (a), we deduce that $b$ lies in the center of $G$, and so $\chi^{\prime}$ lies in $\operatorname{Irr}(G)$. By Proposition 3.2, we must have $\chi(b) \chi^{\prime}(a)=\operatorname{deg} \chi \operatorname{deg} \chi^{\prime}$. Applying condition (b), we get $b=e$ and $\chi^{\prime}=1$, and it follows that $(a, \chi)$ is a generator of $\operatorname{Rep}(D(G))$.

Conversely, suppose that $(a, \chi)$ is a generator of $\operatorname{Rep}(D(G))$. Then the only simple object that centralizes $(a, \chi)$ is the trivial simple object $(e, 1)$. Let $b \in Z(G)$, let $\chi^{\prime} \in \operatorname{Irr}(G)$, and suppose that $\chi(b) \chi^{\prime}(a)=\operatorname{deg} \chi \operatorname{deg} \chi^{\prime}$. Then the simple objects $(a, \chi)$ and $\left(b, \chi^{\prime}\right)$ centralize each other, by Proposition 3.2. Since the only simple object that centralizes $(a, \chi)$ is the trivial simple object $(e, 1)$, it follows that $b=e$ and $\chi^{\prime}=1$, showing that condition (b) holds.

To see that condition (a) holds, let $H$ denote the normal closure of $a$ in $G$, and suppose that $H \neq G$. By Proposition 3.2, for all $\chi^{\prime} \in \operatorname{Irr}(G)$, the simple objects $(a, \chi)$ and $\left(e, \chi^{\prime}\right)$ centralize each other if and only if $a \in \operatorname{Ker} \chi^{\prime}$, equivalently, $H \leq \operatorname{Ker} \chi^{\prime}$. Since $H$ is proper in $G$, the action of $G$ on the coset space $G / H$ is not trivial, and so the corresponding representation contains a nontrivial irreducible constituent; let $\chi^{\prime}$ denote the character of this constituent. Since $H$ is normal in $G$, it acts trivially on $G / H$, and so $H \leq \operatorname{Ker} \chi^{\prime}$. It follows that $(a, \chi)$ and $\left(e, \chi^{\prime}\right)$ centralize each other, a contradiction. Hence $H=G$, showing that condition (a) holds.

Corollary 3.4. Let $G$ be a finite group with trivial center, and let $(a, \chi)$ be a simple object of $\operatorname{Rep}(D(G))$. Then $(a, \chi)$ is a generator of $\operatorname{Rep}(D(G))$ if and only if the normal closure of a in $G$ is equal to $G$.

Proof. Suppose that the normal closure of $a$ in $G$ is equal to $G$. To see that condition (b) of Theorem 3.3 holds, let $\chi^{\prime} \in \operatorname{Irr}(G)$, and suppose that the simple objects $(a, \chi)$ and $\left(e, \chi^{\prime}\right)$ centralize each other. By Proposition 3.2, the element $a$ belongs to $\operatorname{Ker} \chi^{\prime}$. Since $\operatorname{Ker} \chi^{\prime}$ is a normal subgroup of $G$, the supposition forces Ker $\chi^{\prime}=G$, equivalently, $\chi^{\prime}=1$, and so condition (b) of Theorem 3.3 holds. Hence $(a, \chi)$ is a generator of $\operatorname{Rep}(D(G))$.

The converse clearly holds, by Theorem 3.3

Next, we address the twisted case. Of course, the untwisted case above is a special case of the twisted case below, but we find it instructive to treat the untwisted case separately, as in [11] and [12]. We recall the following result from [11].

Proposition 3.5. Let $G$ be a finite group, let $\omega$ be a normalized 3-cocycle on $G$, and let $(a, \chi)$ and $\left(b, \chi^{\prime}\right)$ be simple objects of $\operatorname{Rep}\left(D^{\omega}(G)\right)$. Then $(a, \chi)$ and $\left(b, \chi^{\prime}\right)$ centralize each other if and only if the following two conditions hold.

(a) The conjugacy classes of a and b commute elementwise. 
(b) For all $x, y \in G$,

$$
\begin{aligned}
& \frac{\beta_{a}\left(x, y^{-1} b y\right) \beta_{a}\left(x y^{-1} b y, x^{-1}\right) \beta_{b}\left(y, x^{-1} a x\right) \beta_{b}\left(y x^{-1} a x, y^{-1}\right)}{\beta_{a}\left(x, x^{-1}\right) \beta_{b}\left(y, y^{-1}\right)} \chi\left(x y^{-1} b y x^{-1}\right) \chi^{\prime}\left(y x^{-1} a x y^{-1}\right) \\
& =\operatorname{deg} \chi \operatorname{deg} \chi^{\prime} .
\end{aligned}
$$

We will need the following result from [12].

Lemma 3.6. Let $G$ be a finite group, let $\omega$ be a normalized 3-cocycle on $G$, and let $a, b, x \in G$. If $a b=b a$, then

$$
\frac{\beta_{a}\left(x, x^{-1}\right)}{\beta_{a}(x, b) \beta_{a}\left(x b, x^{-1}\right)}=\frac{\beta_{b}\left(x^{-1}, x\right)}{\beta_{b}\left(x^{-1}, a\right) \beta_{b}\left(x^{-1} a, x\right)} .
$$

Lemma 3.7. Let $G$ be a finite group, and let $\mu$ be a normalized 2-cocycle on $G$.

(a) For all $a, x, y \in G$,

$$
\frac{\mu\left(y, x^{-1} a x\right) \mu\left(y x^{-1} a x, y^{-1}\right) \mu\left(x y^{-1}, y x^{-1} a x y^{-1}\right)}{\mu\left(y, y^{-1}\right) \mu\left(a, x y^{-1}\right)}=\frac{\mu\left(x, x^{-1} a x\right)}{\mu(a, x)} .
$$

(b) For all $a, x \in G$,

$$
\frac{\mu\left(x, x^{-1}\right)}{\mu\left(x^{-1}, a\right) \mu\left(x^{-1} a, x\right)}=\frac{\mu\left(x, x^{-1} a\right)}{\mu\left(x^{-1} a, x\right)}=\frac{\mu\left(x, x^{-1} a x\right)}{\mu(a, x)} .
$$

Proof. That $\mu$ is a normalized 2-cocyle means that

$$
\mu(y, z) \mu(x, y z)=\mu(x y, z) \mu(x, y)
$$

for all $x, y, z \in G$, and $\mu(x, y)=1$ if $x$ or $y$ is the identity element. Applying the 2-cocycle condition of $\mu$ to the triple $\left(x y^{-1}, y x^{-1} a x, y^{-1}\right)$ gives

$\mu\left(y x^{-1} a x, y^{-1}\right) \mu\left(x y^{-1}, y x^{-1} a x y^{-1}\right)=\mu\left(a x, y^{-1}\right) \mu\left(x y^{-1}, y x^{-1} a x\right)$. Making this substitution in the expression

$$
\frac{\mu\left(y, x^{-1} a x\right) \mu\left(y x^{-1} a x, y^{-1}\right) \mu\left(x y^{-1}, y x^{-1} a x y^{-1}\right)}{\mu\left(y, y^{-1}\right) \mu\left(a, x y^{-1}\right)}
$$

yields

$$
\frac{\mu\left(y, x^{-1} a x\right) \mu\left(a x, y^{-1}\right) \mu\left(x y^{-1}, y x^{-1} a x\right)}{\mu\left(y, y^{-1}\right) \mu\left(a, x y^{-1}\right)} .
$$

Applying the 2-cocycle condition of $\mu$ to the triple $\left(a, x, y^{-1}\right)$ gives $\frac{\mu\left(a x, y^{-1}\right)}{\mu\left(a, x y^{-1}\right)}=$ $\frac{\mu\left(x, y^{-1}\right)}{\mu(a, x)}$. Making this substitution in the expression above yields

$$
\frac{\mu\left(y, x^{-1} a x\right) \mu\left(x y^{-1}, y x^{-1} a x\right) \mu\left(x, y^{-1}\right)}{\mu\left(y, y^{-1}\right) \mu(a, x)} .
$$

Applying the 2-cocycle condition of $\mu$ to the triple $\left(y, y^{-1}, y x^{-1} a x\right)$ gives $\frac{\mu\left(y, x^{-1} a x\right)}{\mu\left(y, y^{-1}\right)}=$ $\frac{1}{\mu\left(y^{-1}, y x^{-1} a x\right)}$. Making this substitution in the expression above yields

$$
\frac{\mu\left(x y^{-1}, y x^{-1} a x\right) \mu\left(x, y^{-1}\right)}{\mu(a, x) \mu\left(y^{-1}, y x^{-1} a x\right)} .
$$


Applying the 2-cocycle condition of $\mu$ to the triple $\left(x, y^{-1}, y x^{-1} a x\right)$ gives $\frac{\mu\left(x y^{-1}, y x^{-1} a x\right) \mu\left(x, y^{-1}\right)}{\mu\left(y^{-1}, y x^{-1} a x\right)}=\mu\left(x, x^{-1} a x\right)$. Making this substitution in the expression above yields

$$
\frac{\mu\left(x, x^{-1} a x\right)}{\mu(a, x)},
$$

establishing (a).

Applying the 2-cocycle condition of $\mu$ to the triple $\left(x, x^{-1}, a\right)$ gives $\frac{\mu\left(x, x^{-1}\right)}{\mu\left(x^{-1}, a\right)}=$ $\mu\left(x, x^{-1} a\right)$. Making this substitution in the expression

$$
\frac{\mu\left(x, x^{-1}\right)}{\mu\left(x^{-1}, a\right) \mu\left(x^{-1} a, x\right)}
$$

yields

$$
\frac{\mu\left(x, x^{-1} a\right)}{\mu\left(x^{-1} a, x\right)},
$$

Applying the 2-cocycle condition of $\mu$ to the triple $\left(x, x^{-1} a, x\right)$ gives $\frac{\mu\left(x, x^{-1} a\right)}{\mu\left(x^{-1} a, x\right)}=$ $\frac{\mu\left(x, x^{-1} a x\right)}{\mu(a, x)}$, and so the expression above is equal to

$$
\frac{\mu\left(x, x^{-1} a x\right)}{\mu(a, x)},
$$

establishing (b).

Proposition 3.8. Let $G$ be a finite group, let $\omega$ be a normalized 3-cocycle on $G$, and let $(a, \chi)$ and $\left(b, \chi^{\prime}\right)$ be simple objects of $\operatorname{Rep}\left(D^{\omega}(G)\right)$, where $b$ lies in the center of $G$, so that $\chi^{\prime} \in \operatorname{Irr}_{\beta_{b}}(G)$. Then $(a, \chi)$ and $\left(b, \chi^{\prime}\right)$ centralize each other if and only if the following holds.

(i) $\chi(b) \chi^{\prime}(a)=\operatorname{deg} \chi \operatorname{deg} \chi^{\prime}$

If $b=e$, then the condition above is equivalent to the condition

(i') $a \in \operatorname{Ker} \chi^{\prime}$.

Proof. If $b=e$, then $\chi^{\prime}$ is an ordinary character, and the equality in condition (i) is equivalent to the equality $\chi^{\prime}(a)=\operatorname{deg} \chi^{\prime}$, which is equivalent to condition $\left(\mathrm{i}^{\prime}\right)$.

Suppose that $(a, \chi)$ and $\left(b, \chi^{\prime}\right)$ centralize each other. Putting $x=y=e$ in condition (b) of Proposition 3.5, we get $\chi(b) \chi^{\prime}(a)=\operatorname{deg} \chi \operatorname{deg} \chi^{\prime}$, which is condition (i).

Conversely, suppose that condition (i) holds. Since $b$ lies in the center of $G$, we know that $C_{G}(b)=G, \beta_{b}$ is a 2-cocycle on $G$, and $\chi^{\prime}$ is a $\beta_{b}$-character of $G$. Condition (a) of Proposition 3.5 clearly holds. It remains to show that condition 
(b) of Proposition 3.5 holds. Let $x, y \in G$. Since $b$ lies in the center of $G$, the left-hand side of condition (b) of Proposition 3.5 reduces to

$$
\frac{\beta_{a}(x, b) \beta_{a}\left(x b, x^{-1}\right) \beta_{b}\left(y, x^{-1} a x\right) \beta_{b}\left(y x^{-1} a x, y^{-1}\right)}{\beta_{a}\left(x, x^{-1}\right) \beta_{b}\left(y, y^{-1}\right)} \chi(b) \chi^{\prime}\left(y x^{-1} a x y^{-1}\right) .
$$

Let $\rho: G \rightarrow \mathrm{GL}(V)$ be a projective $\beta_{b}$-representation of $G$ whose character is $\chi^{\prime}$, and let $z \in G$. Then

$$
\rho(a) \rho(z)=\beta_{b}(a, z) \rho(a z)=\beta_{b}(a, z) \rho\left(z\left(z^{-1} a z\right)\right)=\frac{\beta_{b}(a, z)}{\beta_{b}\left(z, z^{-1} a z\right)} \rho(z) \rho\left(z^{-1} a z\right),
$$

and so

$$
\rho\left(z^{-1} a z\right)=\frac{\beta_{b}\left(z, z^{-1} a z\right)}{\beta_{b}(a, z)}[\rho(z)]^{-1} \rho(a) \rho(z) .
$$

Taking the trace of both sides, we get

$$
\chi^{\prime}\left(z^{-1} a z\right)=\frac{\beta_{b}\left(z, z^{-1} a z\right)}{\beta_{b}(a, z)} \chi^{\prime}(a) .
$$

Putting $z=x y^{-1}$ in the equation above, we get

$$
\chi^{\prime}\left(y x^{-1} a x y^{-1}\right)=\frac{\beta_{b}\left(x y^{-1}, y x^{-1} a x y^{-1}\right)}{\beta_{b}\left(a, x y^{-1}\right)} \chi^{\prime}(a) .
$$

Substituting the expression above in (3), we get

$$
\frac{\beta_{a}(x, b) \beta_{a}\left(x b, x^{-1}\right) \beta_{b}\left(y, x^{-1} a x\right) \beta_{b}\left(y x^{-1} a x, y^{-1}\right) \beta_{b}\left(x y^{-1}, y x^{-1} a x y^{-1}\right)}{\beta_{a}\left(x, x^{-1}\right) \beta_{b}\left(y, y^{-1}\right) \beta_{b}\left(a, x y^{-1}\right)} \chi(b) \chi^{\prime}(a) .
$$

Using Lemma 3.6, we see that the expression above is equal to

$$
\frac{\beta_{b}\left(x^{-1}, a\right) \beta_{b}\left(x^{-1} a, x\right) \beta_{b}\left(y, x^{-1} a x\right) \beta_{b}\left(y x^{-1} a x, y^{-1}\right) \beta_{b}\left(x y^{-1}, y x^{-1} a x y^{-1}\right)}{\beta_{b}\left(x^{-1}, x\right) \beta_{b}\left(y, y^{-1}\right) \beta_{b}\left(a, x y^{-1}\right)} \chi(b) \chi^{\prime}(a) \text {. }
$$

Applying Lemma 3.7 with $\mu=\beta_{b}$, and noting that $\beta_{b}\left(x^{-1}, x\right)=\beta_{b}\left(x, x^{-1}\right)$, we see that the expression above reduces to $\chi(b) \chi^{\prime}(a)$. By supposition, $\chi(b) \chi^{\prime}(a)=$ $\operatorname{deg} \chi \operatorname{deg} \chi^{\prime}$, and so condition (b) of Proposition 3.5 holds. Hence $(a, \chi)$ and $\left(b, \chi^{\prime}\right)$ centralize each other.

Theorem 3.9. Let $G$ be a finite group, let $\omega$ be a normalized 3-cocycle on $G$, and let $(a, \chi)$ be a simple object of $\operatorname{Rep}\left(D^{\omega}(G)\right)$. Then $(a, \chi)$ is a generator of $\operatorname{Rep}\left(D^{\omega}(G)\right)$ if and only if the following two conditions hold.

(a) The normal closure of a in $G$ is equal to $G$.

(b) For all $b \in Z(G)$ and $\chi^{\prime} \in \operatorname{Irr}_{\beta_{b}}(G)$, if $\chi(b) \chi^{\prime}(a)=\operatorname{deg} \chi \operatorname{deg} \chi^{\prime}$ (equivalently, $(a, \chi)$ and $\left(b, \chi^{\prime}\right)$ centralize each other $)$, then $b=e$ and $\chi^{\prime}=1$. 
Proof. The proof to be given is almost identical to the one given for the untwisted case. By the double centralizer theorem, the simple object $(a, \chi)$ is a generator of $\operatorname{Rep}\left(D^{\omega}(G)\right)$ if and only if the only simple object that centralizes $(a, \chi)$ is the trivial simple object $(e, 1)$.

Suppose that conditions (a) and (b) in the statement of the theorem hold, and let $\left(b, \chi^{\prime}\right)$ be a simple object of $\operatorname{Rep}\left(D^{\omega}(G)\right)$ that centralizes $(a, \chi)$. By Proposition 3.5 , the conjugacy classes of $a$ and $b$ commute elementwise; combining this fact with condition (a), we deduce that $b$ lies in the center of $G$, and so $\chi^{\prime} \operatorname{lies}$ in $\operatorname{Irr}_{\beta_{b}}(G)$. By Proposition 3.8, we must have $\chi(b) \chi^{\prime}(a)=\operatorname{deg} \chi \operatorname{deg} \chi^{\prime}$. Applying condition (b), we get $b=e$ and $\chi^{\prime}=1$, and it follows that $(a, \chi)$ is a generator of $\operatorname{Rep}\left(D^{\omega}(G)\right)$.

Conversely, suppose that $(a, \chi)$ is a generator of $\operatorname{Rep}\left(D^{\omega}(G)\right)$. Then the only simple object that centralizes $(a, \chi)$ is the trivial simple object $(e, 1)$. Let $b \in Z(G)$, let $\chi^{\prime} \in \operatorname{Irr}_{\beta_{b}}(G)$, and suppose that $\chi(b) \chi^{\prime}(a)=\operatorname{deg} \chi \operatorname{deg} \chi^{\prime}$. Then the simple objects $(a, \chi)$ and $\left(b, \chi^{\prime}\right)$ centralize each other, by Proposition 3.8. Since the only simple object that centralizes $(a, \chi)$ is the trivial simple object $(e, 1)$, it follows that $b=e$ and $\chi^{\prime}=1$, showing that condition (b) holds.

To see that condition (a) holds, let $H$ denote the normal closure of $a$ in $G$, and suppose that $H \neq G$. By Proposition 3.8, for all $\chi^{\prime} \in \operatorname{Irr}(G)$, the simple objects $(a, \chi)$ and $\left(e, \chi^{\prime}\right)$ centralize each other if and only if $a \in \operatorname{Ker} \chi^{\prime}$, equivalently, $H \leq \operatorname{Ker} \chi^{\prime}$. Since $H$ is proper in $G$, the action of $G$ on the coset space $G / H$ is not trivial, and so the corresponding representation contains a nontrivial irreducible constituent; let $\chi^{\prime}$ denote the character of this constituent. Since $H$ is normal in $G$, it acts trivially on $G / H$, and so $H \leq \operatorname{Ker} \chi^{\prime}$. It follows that $(a, \chi)$ and $\left(e, \chi^{\prime}\right)$ centralize each other, a contradiction. Hence $H=G$, showing that condition (a) holds.

Corollary 3.10. Let $G$ be a finite group with trivial center, let $\omega$ be a normalized 3-cocycle on $G$, and let $(a, \chi)$ be a simple object of $\operatorname{Rep}\left(D^{\omega}(G)\right)$. Then $(a, \chi)$ is a generator of $\operatorname{Rep}\left(D^{\omega}(G)\right)$ if and only if the normal closure of a in $G$ is equal to $G$.

Example 3.11. Let $G$ be a finite group, and let $\omega$ be a normalized 3-cocycle on $G$. If $G$ has trivial center, and $a$ is an element of $G$ whose normal closure is $G$, then, by Corollary 3.10, for every irreducible $\beta_{a}$-character of $C_{G}(a)$, the simple object $(a, \chi)$ is a generator of $\operatorname{Rep}\left(D^{\omega}(G)\right)$. We give three related examples below.

(a) Take $G=S_{n}$, the symmetric group on $n$ letters, with $n \geq 3$. Then $S_{n}$ has trivial center, and the normal closure of the transposition $\sigma=(12)$ is $S_{n}$. Therefore, for every irreducible $\beta_{\sigma}$-character of $C_{S_{n}}(\sigma)$, the simple object $(\sigma, \chi)$ is a generator of $\operatorname{Rep}\left(D^{\omega}\left(S_{n}\right)\right)$. 
(b) Let $n \geq 3$ be an odd integer, and take $G=\operatorname{Dih}_{n}$, the dihedral group of order $2 n$ generated by the elements $a$ and $b$ subject to the relations $a^{n}=e$, $b^{2}=e$, and $b a=a^{-1} b$. Then $\operatorname{Dih}_{n}$ has trivial center, and the normal closure of the element $b$ is $\operatorname{Dih}_{n}$. Therefore, for every irreducible $\beta_{b}$-character of $C_{\operatorname{Dih}_{n}}(b)=\{e, b\}$, the simple object $(b, \chi)$ is a generator of $\operatorname{Rep}\left(D^{\omega}\left(\operatorname{Dih}_{n}\right)\right)$. Note that, since the Schur multiplier of a cyclic group is trivial, the 2-cocycle $\beta_{b}$ is cohomologically trivial, and so $\chi$ may be identified with an ordinary character.

(c) Suppose that $G$ is nonabelian and simple. Then $G$ has trivial center, and the normal closure of every nontrivial element $a$ is $G$. Therefore, for every nontrivial element $a$, and for every irreducible $\beta_{a}$-character of $C_{G}(a)$, the simple object $(a, \chi)$ is a generator of $\operatorname{Rep}\left(D^{\omega}(G)\right)$.

Example 3.12. Let $p$ be an odd prime, and consider the special linear group $\mathrm{SL}(2, p)$ consisting of all $2 \times 2$ matrices of determinant 1 whose entries belong to the field of $p$ elements. This group has order $p^{3}-p^{2}$. The matrices

$$
X=\left(\begin{array}{rr}
0 & -1 \\
1 & 0
\end{array}\right) \quad \text { and } \quad Y=\left(\begin{array}{ll}
1 & 1 \\
0 & 1
\end{array}\right)
$$

generate the group $\mathrm{SL}(2, p)$. The center of $\mathrm{SL}(2, p)$ is a subgroup of order 2 , consisting of the matrices $\pm\left(\begin{array}{ll}1 & 0 \\ 0 & 1\end{array}\right)$.

The character table of $\mathrm{SL}(2, p)$ was first obtained by F. G. Frobenius. Later, I. Schur [13] and independently H. Jordan [9] obtained the characters of the special linear groups over arbitrary finite fields [8]. We use the exposition given in [5]. The group $\operatorname{SL}(2, p)$ has exactly $p+4$ distinct irreducible characters. For the purpose of this example, we will only need a portion of the character table of $\operatorname{SL}(2, p)$. Set $\epsilon=(-1)^{(p-1) / 2}$. The table below gives the values of the irreducible characters evaluated at the identity matrix $I$ and at the matrix $Y$, omitting identical columns.

\begin{tabular}{|c||c|c|c|c|c|c|c|c|}
\hline$I$ & 1 & $p$ & $p+1$ & $p-1$ & $\frac{p+1}{2}$ & $\frac{p+1}{2}$ & $\frac{p-1}{2}$ & $\frac{p-1}{2}$ \\
\hline$Y$ & 1 & 0 & 1 & -1 & $\frac{1+\sqrt{\epsilon p}}{2}$ & $\frac{1-\sqrt{\epsilon p}}{2}$ & $\frac{-1+\sqrt{\epsilon p}}{2}$ & $\frac{-1-\sqrt{\epsilon p}}{2}$ \\
\hline
\end{tabular}

It is easily verified that $X=Y^{-1}\left(X Y^{-1} X^{-1}\right) Y^{-1}$, and since the matrices $X$ and $Y$ generate $\mathrm{SL}(2, p)$, it follows that the normal closure of $Y$ is $\mathrm{SL}(2, p)$.

The conjugacy class of $Y$ contains $\left(p^{2}-1\right) / 2$ elements, and so the centralizer of $Y$ in $\operatorname{SL}(2, p)$ has order $2 p$. The matrix $Y$ has order $p$, so the matrix $-Y$ has order $2 p$, and it follows that the centralizer of $Y$ in $\mathrm{SL}(2, p)$ is a cyclic group with generator $-Y$. 
Let $\zeta$ be a primitive $2 p$-th root of unity. For each $1 \leq i \leq 2 p$, let $\chi_{i}:\langle-Y\rangle \rightarrow \mathbb{C}^{\times}$ denote the group homomorphism that sends $-Y$ to $\zeta^{i}$. Then the $\chi_{i}$ constitute all of the irreducible characters of the centralizer of $Y$ in $\operatorname{SL}(2, p)$. We have

$$
\left.\chi_{i}(-I)=\chi_{i}\left((-Y)^{p}\right)\right)=\zeta^{p i}=(-1)^{i} .
$$

Suppose that $i$ is odd. We will show that the simple object $\left(Y, \chi_{i}\right)$ is a generator of $\operatorname{Rep}(D(\operatorname{SL}(2, p)))$. We have shown above that condition (a) of Theorem 3.3. To see that condition (b) of Theorem 3.3 holds, let $\chi^{\prime}$ be an irreducible character of $\mathrm{SL}(2, p)$. Suppose that the simple objects $\left(Y, \chi_{i}\right)$ and $\left(I, \chi^{\prime}\right)$ centralize each other. Then $Y \in \operatorname{Ker} \chi^{\prime}$, by Proposition 3.2. Since the normal closure of $Y$ is $\mathrm{SL}(2, p)$, it follows that $\chi^{\prime}=1$. We have

$$
\chi_{i}(-I) \chi^{\prime}(Y)=-\chi^{\prime}(Y)
$$

and

$$
\operatorname{deg} \chi_{i} \operatorname{deg} \chi^{\prime}=\operatorname{deg} \chi^{\prime} .
$$

Inspecting the partial character table given above, we see that $-\chi^{\prime}(Y) \neq \operatorname{deg} \chi^{\prime}$, and so the simple objects $\left(Y, \chi_{i}\right)$ and $\left(-I, \chi^{\prime}\right)$ do not centralize each other, by Proposition 3.2. It follows that condition (b) of Theorem 3.3 also holds, proving that $\left(Y, \chi_{i}\right)$ is a generator of $\operatorname{Rep}(D(\operatorname{SL}(2, p)))$.

Note that if $i$ is even, then the simple objects $\left(Y, \chi_{i}\right)$ and $(-I, 1)$ centralize each other, and so $\left(Y, \chi_{i}\right)$ is not a generator of $\operatorname{Rep}(D(\operatorname{SL}(2, p)))$, by Theorem 3.3.

\section{Adjoint category}

In this section, we describe the adjoint category of $\operatorname{Rep}\left(D^{\omega}(G)\right)$. The case where $\omega=1$ was addressed in the paper [12]. For a fusion category $\mathcal{C}$, adjoint category of $\mathcal{C}$, denoted $\mathcal{C}_{\text {ad }}$, is the full fusion subcategory of $\mathcal{C}$ generated by all subobjects of $X \otimes X^{*}$, where $X$ runs through simple objects of $\mathcal{C}$. For example, for a finite group $G$, we have $\operatorname{Rep}(G)_{\text {ad }} \cong \operatorname{Rep}(G / Z(G))$.

Lemma 4.1. Let $G$ be a finite group, and let $\omega$ be a normalized 3-cocycle on $G$.

The set

$$
Z_{\omega}(G)=\left\{a \in Z(G) \mid \beta_{a} \text { is cohomologically trivial }\right\}
$$

is a subgroup of $Z(G)$.

Proof. Since $\beta_{e}=1$, the identity element $e$ lies in $Z_{\omega}(G)$. Let $a, b \in Z_{\omega}(G)$. Define a function $\tau_{a, b}: G \rightarrow \mathbb{C}^{\times}$by $\tau_{a, b}(x)=\beta_{x}(a, b)$. It follows from (2) that

$$
\beta_{a b}=\beta_{a} \cdot \beta_{b} \cdot d \tau_{a, b},
$$


showing that $\beta_{a b}$ and $\beta_{a} \beta_{b}$ are cohomologous, where $d$ denotes the coboundary operator. Since $\beta_{a}$ and $\beta_{b}$ are cohomologically trivial, the same is true for $\beta_{a b}$, and so $a b \in Z_{\omega}(G)$.

The following definition is taken from [12].

Definition 4.2. Let $G$ be a finite group, let $\omega$ be a normalized 3-cocycle on $G$, let $K$ and $H$ be normal subgroups of $G$ that commute elementwise, and let $B$ : $K \times H \rightarrow \mathbb{C}^{\times}$be a function. We say that $B$ is an $\omega$-bicharacter on $K \times H$ if

(a) $B(x, u v)=\beta_{x}^{-1}(u, v) B(x, u) B(x, v)$, and

(b) $B(x y, u)=\beta_{u}(x, y) B(x, u) B(y, u)$

for all $x, y \in K$ and $u, v \in H$. We say that $B$ is $G$-invariant if

$$
B\left(g^{-1} x g, u\right)=\frac{\beta_{x}(g, u) \beta_{x}\left(g u, g^{-1}\right)}{\beta_{x}\left(g, g^{-1}\right)} B\left(x, g u g^{-1}\right)
$$

for all $g \in G, x \in K$, and $u \in H$.

We refer the reader to [12] for an explanation of the $G$-invariance property and the apparent lack of symmetry. It was shown in [12] that the fusion subcategories of $\operatorname{Rep}\left(D^{\omega}(G)\right)$ are parametrized by triples $(K, H, B)$, where $K$ and $H$ are normal subgroups of $G$ that commute elementwise, and $B$ is an $\omega$-bicharacter on $K \times H$. Given such a triple $(K, H, B)$, denote by $\mathcal{S}(K, H, B)$ the full abelian subcategory generated by simple objects $(a, \chi)$ such that $a \in K$ and $\chi(h)=B(a, h) \operatorname{deg} \chi$ for all $h \in H$. It was shown in [12] that $\mathcal{S}(K, H, B)$ is, in fact, a fusion subcategory of $\operatorname{Rep}\left(D^{\omega}(G)\right)$, and

$$
\mathcal{S}(K, H, B)^{\prime}=\mathcal{S}\left(H, K,\left(B^{\mathrm{op}}\right)^{-1}\right)
$$

where $\left(B^{\text {op }}\right)^{-1}: H \times K \rightarrow \mathbb{C}^{\times}$is defined by $\left(B^{\text {op }}\right)^{-1}(h, k)=B(k, h)^{-1}$.

Lemma 4.3. Let $G$ be a finite group, and let $\omega$ be a normalized 3-cocycle on $G$.

(a) For all $a, g, x, y \in G$,

$$
\begin{aligned}
\beta_{a}\left(g x g^{-1}, g y g^{-1}\right) & \cdot \frac{\beta_{a}\left(g, g^{-1}\right)}{\beta_{a}(g, x y) \beta_{a}\left(g x y, g^{-1}\right)} \\
& =\beta_{g^{-1} a g}(x, y) \cdot \frac{\beta_{a}\left(g, g^{-1}\right)}{\beta_{a}(g, x) \beta_{a}\left(g x, g^{-1}\right)} \cdot \frac{\beta_{a}\left(g, g^{-1}\right)}{\beta_{a}(g, y) \beta_{a}\left(g y, g^{-1}\right)} .
\end{aligned}
$$

(b) For all $a, g, x, y \in G$, if a lies in $Z_{\omega}(G)$, then

$$
\beta_{a}\left(g x g^{-1}, g y g^{-1}\right)=\beta_{a}(x, y)
$$


Proof. Part (a) was proved in [12]. To see (b), let $g, x, y \in G$, and let $a \in Z_{\omega}(G)$. Applying Lemma 3.7 with $\mu=\beta_{a}$ to the equality in (a), we get

$$
\beta_{a}\left(g x g^{-1}, g y g^{-1}\right) \cdot \frac{\beta_{a}\left(g^{-1}, g x y\right)}{\beta_{a}\left(g x y, g^{-1}\right)}=\beta_{a}(x, y) \cdot \frac{\beta_{a}\left(g^{-1}, g x\right)}{\beta_{a}\left(g x, g^{-1}\right)} \cdot \frac{\beta_{a}\left(g^{-1}, g y\right)}{\beta_{a}\left(g y, g^{-1}\right)} .
$$

Since $\beta_{a}$ is cohomologically trivial, it is symmetric, and so the equation above reduces to

$$
\beta_{a}\left(g x g^{-1}, g y g^{-1}\right)=\beta_{a}(x, y),
$$

proving (b).

Lemma 4.4. Let $G$ be a finite group, let $\omega$ be a normalized 3-cocycle on $G$, let $K$ be a subgroup of $Z_{\omega}(G)$, and let $H$ be a subgroup of the commutator subgroup $[G, G]$ of $G$. For each $a \in K$, choose a function $\sigma_{a}: G \rightarrow \mathbb{C}^{\times}$such that $d \sigma_{a}=\beta_{a}$. The function $B: K \times H \rightarrow \mathbb{C}^{\times}$defined by $B(a, x)=\sigma_{a}(x)$ does not depend on the choice of the $\sigma_{a}$, and it is a $G$-invariant $\omega$-bicharacter on $K \times H$.

Proof. Since the restriction of any homomorphism $G \rightarrow \mathbb{C}^{\times}$to the subgroup $H$ is trivial, we deduce that for any two functions $f_{1}: G \rightarrow \mathbb{C}^{\times}$and $f_{2}: G \rightarrow \mathbb{C}^{\times}$, if $d f_{1}=d f_{2}$, then $f_{1}$ and $f_{2}$ are equal when restricted to $H$. It follows that the function $B$ does not depend on the choice of the $\sigma_{a}$.

The condition $d \sigma_{a}=\beta_{a}$ is equivalent to the first condition in the definition of $\omega$-character. To see that the second condition in the definition of $\omega$-character holds, let $a, b \in K$. Define a function $\tau_{a, b}: G \rightarrow \mathbb{C}^{\times}$by $\tau_{a, b}(x)=\beta_{x}(a, b)$. As seen in the proof of Lemma 4.1,

$$
\beta_{a b}=\beta_{a} \cdot \beta_{b} \cdot d \tau_{a, b}=d\left(\sigma_{a} \cdot \sigma_{b} \cdot \tau_{a, b}\right) .
$$

Since we also have $\beta_{a b}=d \sigma_{a b}$, we deduce that the functions $\sigma_{a b}$ and $\sigma_{a} \cdot \sigma_{b} \cdot \tau_{a, b}$ are equal when restricted to $H$, that is, for all $x \in H$,

$$
\sigma_{a b}(x)=\sigma_{a}(x) \sigma_{b}(x) \tau_{a, b}(x),
$$

equivalently,

$$
B(a b, x)=\beta_{x}(a, b) B(a, x) B(b, x),
$$

which is the second condition in the definition of $\omega$-character.

To see that $B$ is $G$-invariant, let $g \in G$, let $a \in K$, and let $x \in H$. Applying the definition of $B$ and Lemma 3.7 with $\mu=\beta_{a}$ to the expression

$$
\frac{\beta_{a}(g, x) \beta_{a}\left(g x, g^{-1}\right)}{\beta_{a}\left(g, g^{-1}\right)} B\left(a, g x g^{-1}\right),
$$


we get

$$
\frac{\beta_{a}\left(g^{-1}, g x\right)}{\beta_{a}\left(g x, g^{-1}\right)} \sigma_{a}\left(g x g^{-1}\right) .
$$

Since $\beta_{a}$ is cohomologically trivial, it is symmetric, and so the expression above reduces to $\sigma_{a}\left(g x g^{-1}\right)$. By Lemma $4.3,\left(\beta_{a}\right)^{g}=\beta_{a}$, and so $d\left(\sigma_{a}\right)^{g}=\left(\beta_{a}\right)^{g}=\beta_{a}=$ $d \sigma_{a}$, where the superscript denotes the conjugation action. Therefore, the functions $\left(\sigma_{a}\right)^{g}$ and $\sigma_{a}$ are equal when restricted to $H$, and so $\sigma_{a}\left(g x g^{-1}\right)=\sigma_{a}(x)=B(a, b)$, proving that $B$ is $G$-invariant.

A fusion category $\mathcal{C}$ is said to be pseudounitary if its categorical dimension coincides with its Frobenius-Perron dimension [6]. In this case, $\mathcal{C}$ admits a canonical spherical structure with respect to which categorical dimensions of objects coincide with their Frobenius-Perron dimensions [6]. The category $\operatorname{Rep}\left(D^{\omega}(G)\right)$ is pseudounitary. In the paper [7], S. Gelaki and D. Nikshych showed that, for a pseudounitary modular category $\mathcal{C}$, the adjoint subcategory $\mathcal{C}_{a d}$ and the full maximal pointed subcategory $\mathcal{C}_{\mathrm{pt}}$ are centralizers of each other, that is,

$$
\mathcal{C}_{a d}=\left(\mathcal{C}_{\mathrm{pt}}\right)^{\prime}
$$

Theorem 4.5. Let $G$ be a finite group, and let $\omega$ be a normalized 3-cocycle on $G$. Then

$$
\operatorname{Rep}\left(D^{\omega}(G)\right)_{\mathrm{pt}}=\mathcal{S}\left(Z_{\omega}(G),[G, G], B\right)
$$

and

$$
\operatorname{Rep}\left(D^{\omega}(G)\right)_{\mathrm{ad}}=\mathcal{S}\left([G, G], Z_{\omega}(G),\left(B^{\mathrm{op}}\right)^{-1}\right)
$$

where $B: Z_{\omega}(G) \times[G, G] \rightarrow \mathbb{C}^{\times}$is the $G$-invariant $\omega$-bicharacter defined in Lemma 4.4 .

Proof. The dimension of a simple object $(a, \chi)$ of $\operatorname{Rep}\left(D^{\omega}(G)\right)$ is $\frac{|G|}{\left|C_{G}(a)\right|} \operatorname{deg} \chi$, which is equal to 1 if and only if $a$ lies in the center of $G$ and $\operatorname{deg} \chi=1$. The latter condition implies that $\beta_{a}$ is cohomologically trivial. It follows that $\operatorname{Rep}\left(D^{\omega}(G)\right)_{\mathrm{pt}}=$ $\mathcal{S}\left(Z_{\omega}(G),[G, G], B\right)$. Applying (4) and (5) to the previous equation, we get $\operatorname{Rep}\left(D^{\omega}(G)\right)_{\mathrm{ad}}=\mathcal{S}\left([G, G], Z_{\omega}(G),\left(B^{\mathrm{op}}\right)^{-1}\right)$.

\section{References}

[1] B. Bakalov and A. Kirillov Jr., Lectures on Tensor Categories and Modular Functors, University Lecture Series, 21, American Mathematical Society, Providence, RI, 2001.

[2] A. Coste, T. Gannon and P. Ruelle, Finite group modular data, Nuclear Phys. B, 581(3) (2000), 679-717. 
[3] R. Dijkgraaf, V. Pasquier and P. Roche, Quasi-quantum groups related to orbifold models, Modern quantum field theory (Bombay, 1990), World Sci. Publ., River Edge, NJ, (1991), 375-383.

[4] R. Dijkgraaf, V. Pasquier and P. Roche, Quasi-Hopf algebras, group cohomology, and orbifold models, Integrable systems and quantum groups (Pavia, 1990), World Sci. Publ., River Edge, NJ, (1992), 75-98.

[5] L. Dornhoff, Group Representation Theory, Part A, M. Dekker (1971).

[6] P. Etingof, D. Nikshych and V. Ostrik, On fusion categories, Ann. of Math. (2), 162 (2005), 581-642.

[7] S. Gelaki and D. Nikshych, Nilpotent fusion categories, Adv. Math., 217 (2008), 1053-1071.

[8] J. E. Humphreys, Representation of SL(2,p), Amer. Math. Monthly, 82 (1975), no. 1, 21-39.

[9] H. Jordan, Group characters of various types of linear groups, Amer. J. Math., 29 (1907), 387-405.

[10] M. Müger, On the structure of modular categories, Proc. London Math. Soc., 87(2) (2003), 291-308.

[11] D. Naidu and D. Nikshych, Lagrangian subcategories and braided tensor equivalences of twisted quantum doubles of finite groups, Comm. Math. Phys., 279 (2008), 845-872.

[12] D. Naidu, D. Nikshych and S. Witherspoon, Fusion subcategories of representation categories of twisted quantum doubles of finite groups, Int. Math. Res. Not. IMRN, 22 (2009), 4183-4219.

[13] I. Schur, Untersuchungen über die Darstellungen der endlichen Gruppen durch gebrochene lineare Substitutionen, J. Reine Angew. Math., 132 (1907) 85-137.

\section{Deepak Naidu}

Department of Mathematical Sciences

Northern Illinois University

DeKalb, Illinois 60115, USA

email: dnaidu@math.niu.edu 